\title{
Research on Fibiril-Reinforced Poroelastic Mechanical Behaviour of Articular Cartilage
}

\author{
Chun LONG ${ }^{1}$, Chang-Yong DOU ${ }^{1}$ and Tong-Tong GUO ${ }^{*}$ \\ ${ }^{1}$ Nature Science Department, Harbin Institute of Technology, (Shenzhen), 518055, Shenzhen, China \\ *tongtong.guo@hitsz.edu.cn
}

Keyword: Biomechanics, Articular cartilage, Porous elasticity, Fibiril-reinforced, Inversion.

\begin{abstract}
The main purpose of this paper is to validate the spring-based fibiril-reinforced porous elastic model of articular cartilage. In this study, the ability to interpret the process of axial reaction and lateral displacement in unconfined compression experiments was used as a criterion to prove the correctness of the model. The parameters of mechanical properties are obtained by means of parameter inversion, and the model is tested based on these parameters. The simulation results and experimental data were compared and obtained: The fibiril-reinforced porous elastic model based on spring can't reflect the stress distribution in the unconfined compression experiment and can't explain the relaxation process of the axial reaction force. This is because the fiber distribution of this model does not match the actual situation, not taking into account the articular cartilage itself inherent viscoelasticity.
\end{abstract}

\section{Introduction}

Osteoarthritis is a common joint disease. Researchers generally believe that osteoarthritis is caused by mechanical overload, so we hope to study the mechanics of the pathogenesis of arthritis. The finite element method is the most effective tool for analyzing joint stress-strain states. In order to carry out the finite element analysis, we first need to establish the joint parts of the finite element model, and which the material properties of articular cartilage is the most complex. Therefore, the use of a good model to reflect the mechanical properties of articular cartilage is extremely important.

Dilsivestro et al. performed unconfined compression experiments of articular cartilage at 2001[1,2]. The newly developed models both interpret the ability of simultaneous processes of axial reaction and lateral displacement in unconfined compression experiments as a criterion to justify their correctness. Wilson et al. (2004) developed a fibiril-reinforced porous viscoelastic model, which compared the results with those of Dilsivestro et al. The model can simultaneously explain these two phenomena [3].
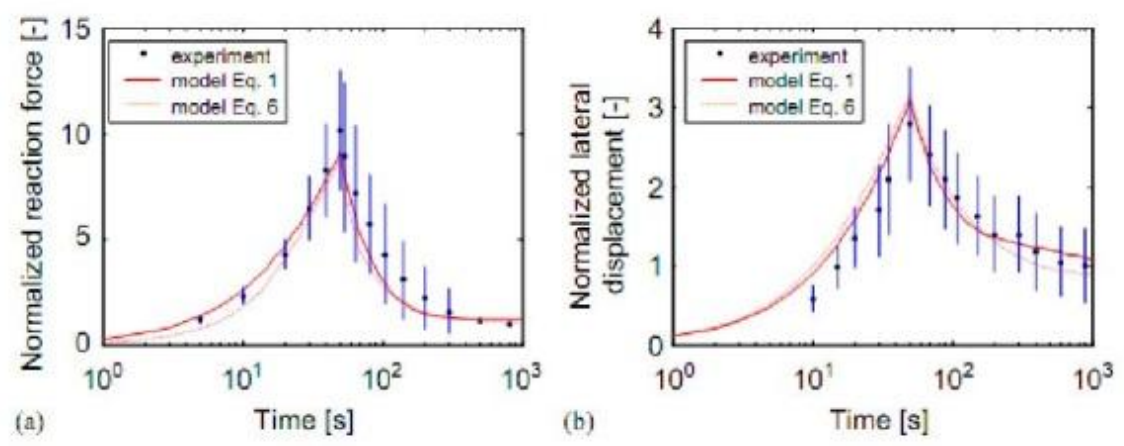

Figure 1. The fitting of the fibiril-reinforced porous viscoelastic model and the experimental data.

Li et al. Proposed a spring-based articular cartilage fibiril-reinforced model in $1999^{[4]}$. No one has so far compared this model with Dilsivestro et al.'s results to determine whether they can explain both phenomenon. In this study, we use this set of experimental data to validate the Li model. 


\section{Methodology}

In this paper, the optimized inversion method developed by Lei et al is introduced and the corresponding material parameters are retrieved from the experimental data of Dilvestro et al., and then verify that the spring-based fibiril-reinforced porous elastic model can simultaneously explain the relaxation of axial reaction force and lateral displacement of the process of change.

\section{Fibiril-Reinforced Model Based on Spring}

The spring-based fibiril-reinforced model was proposed by $\mathrm{Li}$ in 1999 on the basis of the fibiril-reinforced model, which simulates fibiril-reinforced with springs. Its constitutive relation is similar to that of the fibiril-reinforced model, and its effective stress is:

$$
\sigma_{E, i}=\sigma_{m, i}+\sigma_{f, i}
$$

$\sigma_{\mathrm{m}, \mathrm{i}}$ and $\sigma_{\mathrm{f}, \mathrm{i}}$ represent the normal stress in the $\mathrm{i}$ direction of the non-fiber portion and the fiber portion, respectively.

In this model, the non-fiber part is still linear elastic, the fiber part of a linear elastic modulus of $E_{0}$ in parallel with a linear elastic modulus of $E_{\varepsilon} \varepsilon_{f}$ nonlinear spring, the mechanical model shown in Figure.

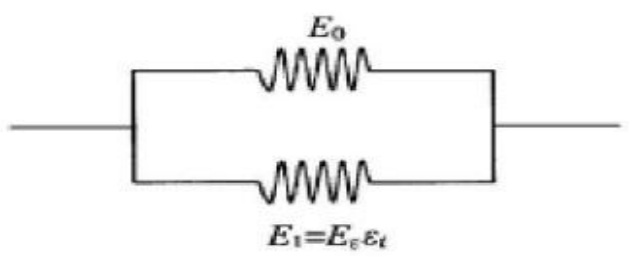

Figure 2. Schematic diagram of elastic fiber.

Elastic modulus of the equation is:

$$
E_{f}=E_{0}+E_{\varepsilon} \varepsilon_{f}
$$

Where $E_{0}, E_{\varepsilon}$---are the initial elastic modulus of the fiber and tensile modulus (Mpa).

In order to express the fiber in Abaqus with spring, the spring stiffness is:

$$
K=2 \pi h E_{f}
$$

The stiffness is distributed over a long spring and four short springs. In order to produce uniform stress under uniform strain, the long spring is subjected to $2 / 3$ of the total stiffness and the other springs are $1 / 6$ of the total stiffness. The elements of the spring-based fibiril-reinforced model are shown in Figure 3.

\section{Geometric Modeling and Meshing}

For the articular cartilage geometry model, this study uses a uniform full-height model. The schematic diagram shown in Figure 3 [5].

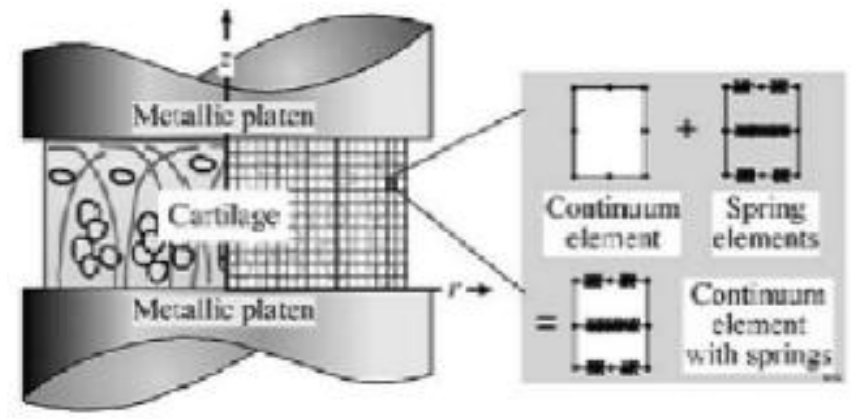

Figure 3. Sketch of fibiril-reinforced model based on spring. 
The geometrical model is established by Abaqus/CAE, and then the mesh is divided into mesh, which consists of 405 nodes and 120 two-dimensional porous elastic elements. The element type is an eight-node axisymmetric element containing pore pressure degrees of freedom, the CAX8P. The geometric model is as follows:

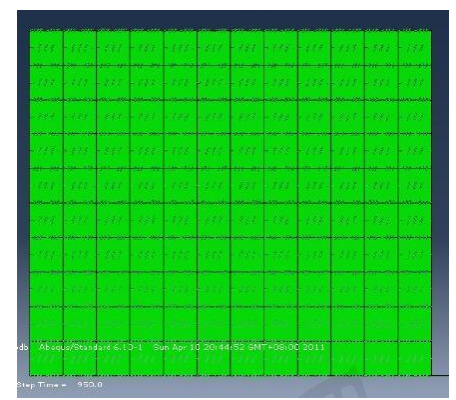

Figure 4. Geometric model of articular cartilage.

The upper and lower indenter are rigid body, the model can be used to analyze rigid body modeling.

\section{Material Properties}

\section{Linear Elastic Isotropic Solid Matrix}

The elastic modulus and the Poisson's ratio can be input under the condition of linear elastic isotropy. In order to conform to the experimental results in this paper, the elastic modulus and Poisson's ratio are, $E_{m}=0.55 \mathrm{Mpa}, V_{m}=0.24$.

\section{Permeability}

Permeability is one of the main parameters of the biphasic porous elastic problem. Using the expansion relationship, the nonlinear permeability can be written as a function of the current porositye, the initial porosity $e_{0}$ :

$$
k=k_{0}\left(\frac{1+e}{1+e_{0}}\right)^{M}
$$

\section{Nonlinear Spring}

In the present model, the fibiril-reinforced of the articular cartilage is achieved by adding a spring element between the nodes. In Abaqus, the nonlinear spring is given by force-relative displacement, and the definition of the nonlinear spring can be seen in Fig 5.

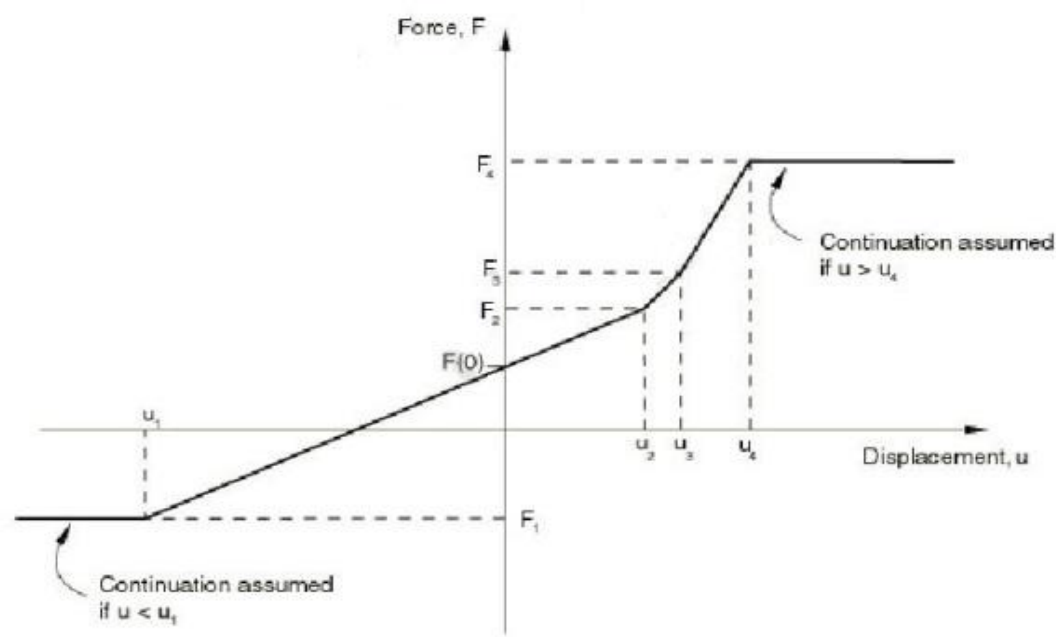

Figure 5. The Definition of Nonlinear Spring in Abaqus. 
The non-linearity of the spring in this paper is approximated by piecewise linearity. The contact conditions of the articular cartilage specimen with the upper and lower indenter were all frictionless.

\section{Load and Boundary Conditions}

The analysis steps and boundary conditions are set as follows. First, set up an analytical step to establish the contact relationship with the following conditions: a fixed indenter; limit the degree of freedom in the r-direction of all nodes on the cartilaginous axis of symmetry; the upper pressure head is moved down to the contact relationship between the cartilage sample and the indenter. The second analysis step is to move the indenter down to $10 \%$ deformation and define the zero pore pressure boundary at the node $r=r 0$. The third analysis step is a relaxation step, which is long enough for the interstitial fluid to flow out of the cartilage matrix and the pressure difference in the matrix disappears and reaches the equilibrium state. The fourth analysis step is a loading analysis step with $5 \%$ deformation at a deformation rate of $0.001 / \mathrm{s}$ and starts to record the reaction force and the lateral displacement. The fifth analysis step is also a relaxation analysis step, so that the cartilage matrix and external force to reach equilibrium again.

After the analysis step and the boundary conditions are defined, the initial porosity defined by the subroutine VOIDRI needs to be assigned to the articular cartilage in the inp file. In order to easily modify the value of the material parameter in the subsequent parameter inversion, we need to parameterize the generated inp file.

\section{Parametric Analysis}

Before parameter inversion, it is necessary to carry out parametric analysis, determine the sensitivity of each parameter, determine the parameters to be used in the optimization process, and give different parameters corresponding to the impact factor. Respectively, $E_{m}, v_{m}, E_{0}, E_{\varepsilon}, k_{0}, M$ parameter analysis. Table 1 gives the reference values for the parameters to be analyzed and the large and small values used for comparison.

Table 1. Parameter values for parameter analysis.

\begin{tabular}{clllll}
\hline $\begin{array}{l}\text { parameter } \\
\text { name }\end{array}$ & $\begin{array}{l}\text { Reference } \\
\text { value }\end{array}$ & $\begin{array}{l}\text { The increased } \\
\text { parameter value }\end{array}$ & $\begin{array}{l}\text { Percentage of } \\
\text { added value to } \\
\text { initial value }(\%)\end{array}$ & $\begin{array}{l}\text { The reduced } \\
\text { parameter } \\
\text { value }\end{array}$ & $\begin{array}{l}\text { Percentage of } \\
\text { reduced value to } \\
\text { initial value }(\%)\end{array}$ \\
\hline$E_{m}(\mathrm{Mpa})$ & 0.55 & 1.65 & 300 & 0.11 & 20 \\
\hline$v_{m}$ & 0.24 & 0.36 & 150 & 0.12 & 502 \\
\hline$E_{0}$ & 1200 & 2400 & 200 & 400 & 33.3 \\
\hline$E_{\varepsilon}$ & 3 & 6 & 200 & 1 & 33.3 \\
\hline$k_{0}$ & $1.71 \mathrm{e}-8$ & $1.71 \mathrm{e}-7$ & 1000 & $1.71 \mathrm{e}-9$ & 10 \\
\hline$M$ & 7 & 10 & 143 & 3 & 43 \\
\hline
\end{tabular}

\section{Inversion Process}

In this study, the parameter inversion was prepared by the optimized inversion method. The first is the objective function and the optimization algorithm. The parameters are determined by parametric analysis to obtain the objective function. As for the choice of optimization algorithm, this paper uses Matlab "lsqnonlin" to solve this nonlinear least squares problem.

\section{Results}

\section{Finite Element Model Validation}

In order to understand the role of fibiril-reinforced and non-linear permeability in unconfined compression experiments in the fibiril-reinforced porous elastic model, the axial reaction and lateral displacement curves simulated by this model are compared with the linear elastic porous elastic 
model and the nonlinear Permeability porous elastic model of the simulation results were compared, as shown in Figure 6,7.

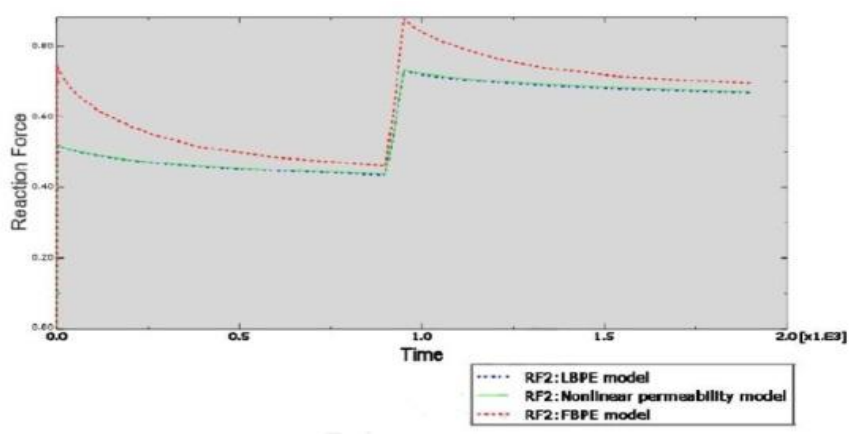

Figure 6. The change process of axial reaction force.

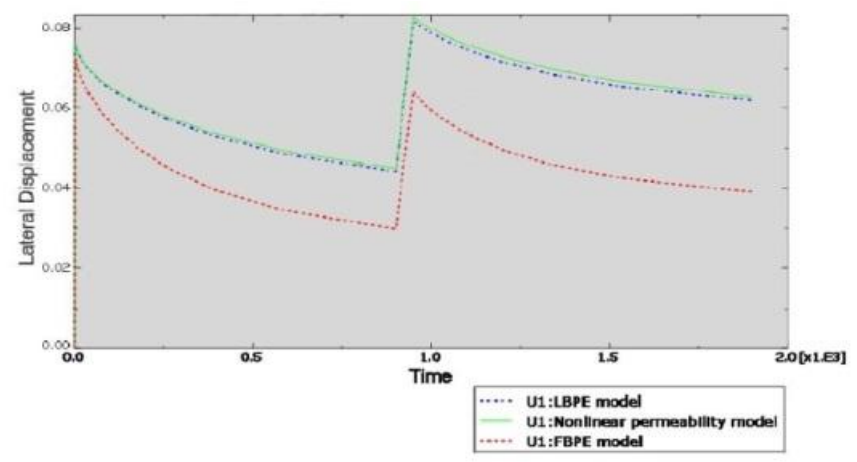

Figure 7. The change process of lateral displacement.

After the simulation, it is necessary to analyze the deformation, pore pressure and stress distribution of the cartilage matrix, the distribution of which is shown in Figure 8,9,10.

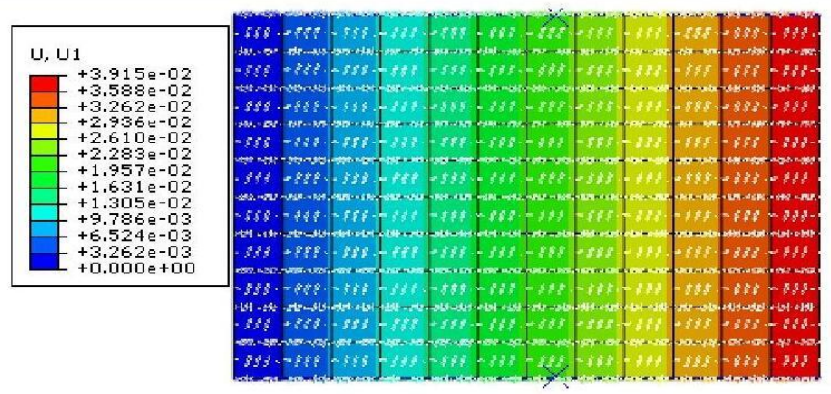

Figure 8. Displacement diagram of equilibrium state.

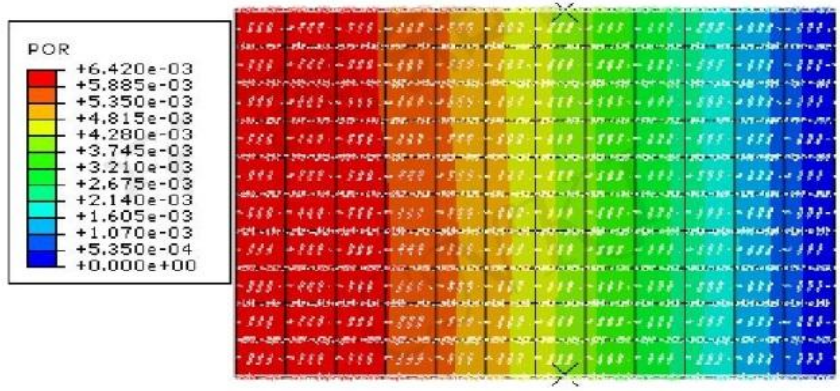

Figure 9. Pore Pressure Distribution in Equilibrium State. 


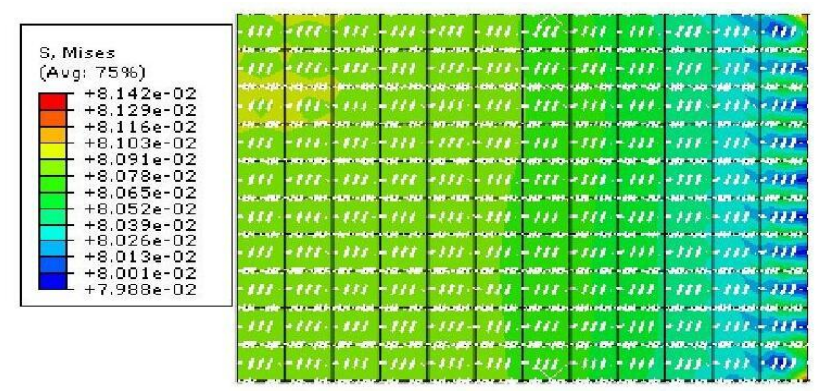

Figure 10. Mises Stress cloud image in equilibrium state.

\section{Parameter Inversion}

The parameters of $E_{m}, v_{m}, E_{0}, E_{\varepsilon}, k_{0}, M$, were analyzed respectively. For each analysis, there is a reference curve, which is used to highlight the output change caused by the change in the parameter value, and then both a high value and a low value are used to observe the model response. The resulting axial reaction force and lateral displacement curve, As shown in Figure 11-15.
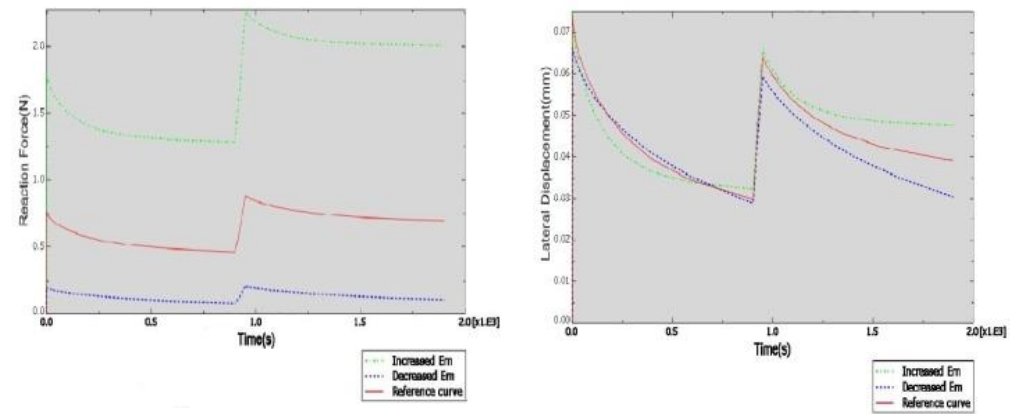

Figure 11. Parametric Analysis of Elastic Modulus of Solid Matrix for Axial Reaction and Lateral Force.
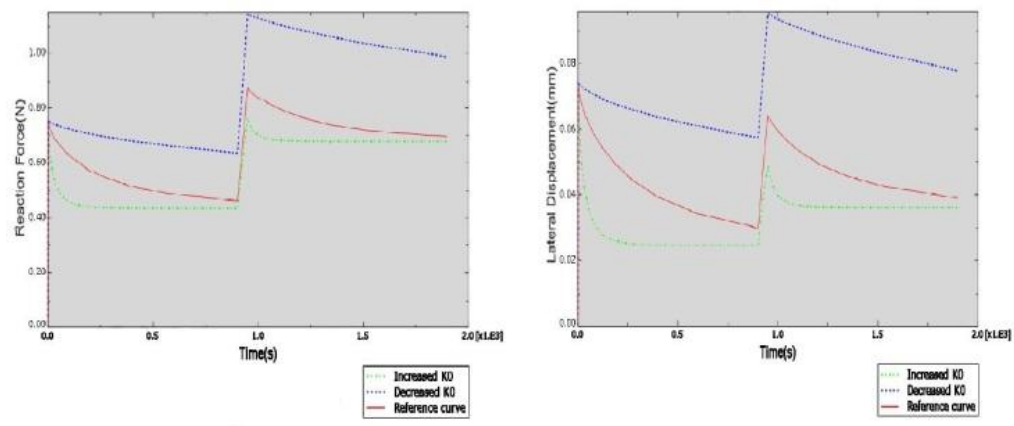

Figure 12. Parametric Analysis of Initial Permeability on Axial Reaction and Lateral Force
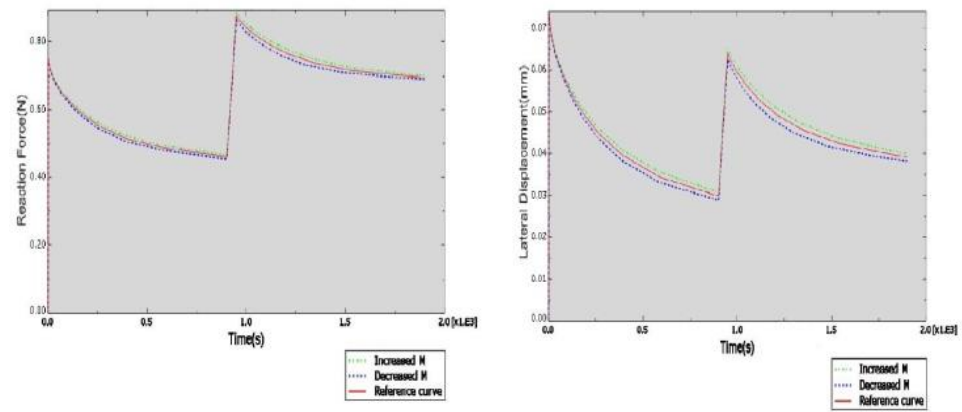

Figure 13. Parametric Analysis of Strain - dependent Permeability on Axial Reaction and Lateral Force 

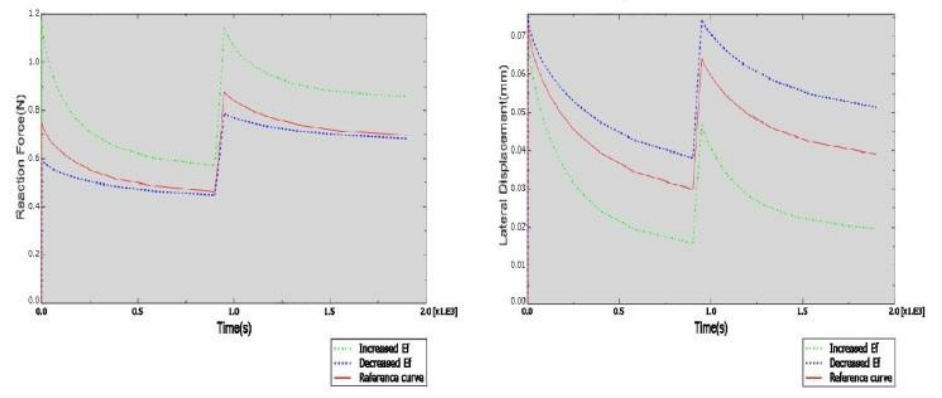

Figure 14. Parametric Analysis of Axial Reaction and Lateral Force in Fiber Mesh Model
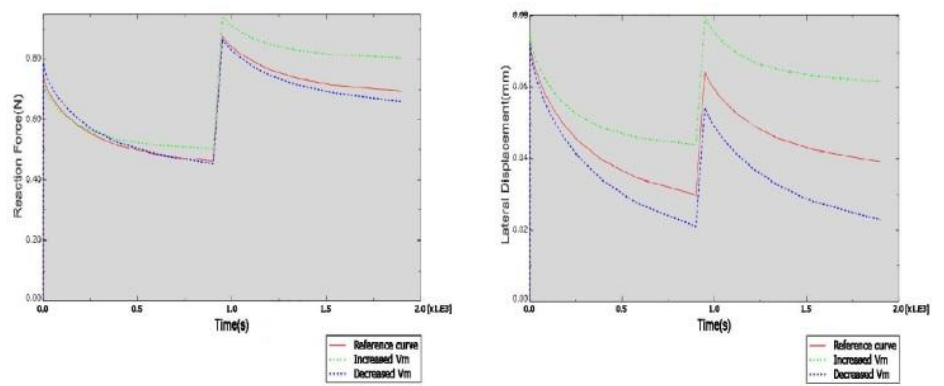

Figure 15. Parametric Analysis of Axial Reaction and Lateral Force in Poisson's Ratio.

The simulated lateral displacements and the experimental data are not different from each other. The parameter values can be retrieved from the lateral displacements. After adjusting the parameters and simulating, the variance of the simulated and experimental values can be calculated $E_{m}=$ $0.55 \mathrm{Mpa}, v_{m}=0.27, E_{0}=3600 \mathrm{Mpa}, E_{\varepsilon}=9 \mathrm{Mpa}, k_{0}=0.9 \times 10^{-7} \mathrm{~mm} / \mathrm{s}, M=7$. The objective function value of 0.96 , this time although the optimal solution may not be obtained, but the error has been small enough, you can use this group of parameter values. The simulation results of the axial reaction force are shown in:

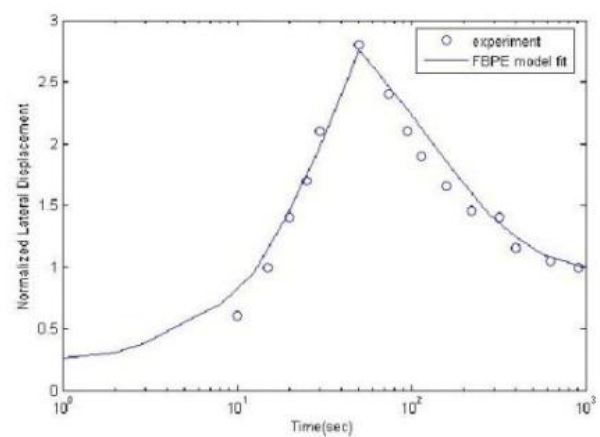

Figure 16. The fibiril-reinforced model was deduced from the lateral displacement.

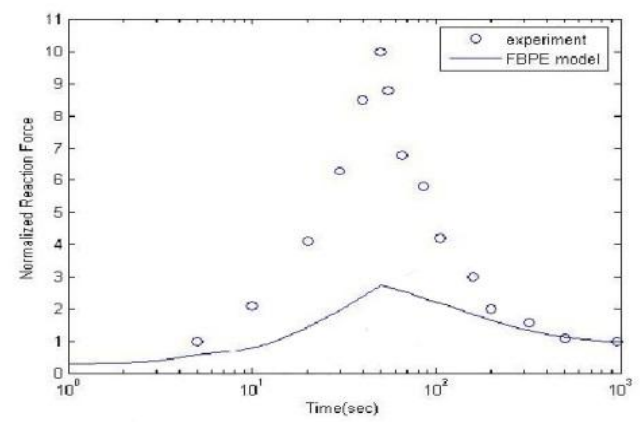

Figure 17. Comparison of Predictive Value and Experimental Value of Axial Reaction Force. 


\section{Discussion}

Model accuracy and computational cost is a contradiction, so often need to trade-off between these two and make a choice. Considering the complexity of the articular cartilage mechanics model and its difficulty in implementation and our existing conditions, this study attempts to study the relative simplicity of the spring-based fibiril-reinforced porous elastic model, hoping to use this model The finite element simulation of the knee joint was performed.

In the finite element simulation, the fibiril-reinforced porous elastic model has some problems in explaining the axial reaction force, but it can explain the basic reflection of the lateral displacement. Only from the reference value point of view, it is necessary to further study the parameters through the parameters of the simulation analysis, we can see that the elastic modulus of the cartilage matrix mainly affects the value of axial reaction force; initial permeability mainly affects the axis The elastic modulus of the fiber mesh has a great influence on the ratio of the peak value of the axial reaction force to the equilibrium state and the value of the lateral displacement. The Poisson's ratio also affects the lateral force And the influence of strain-related permeability on both can be neglected.

The results show that the axial reaction force and lateral displacement are significantly affected by the fibiril-reinforced on the unconfined compression experiment. The effect of the fiber reaction on the axial reaction force is mainly due to the higher peak value and the balance value of the linear elastic porous elastic model, The relaxation behavior is more obvious, and it can be seen that although the value of the axial reaction force in the equilibrium state increases little in the fibiril-reinforced model, the value of the axial reaction force is still increasing; the influence on the lateral displacement is mainly manifested in It is easy to understand that the reinforcement of the fiber is resistant to lateral stretching and the lateral displacement is reduced. This is consistent with the description in the literature [6,7].

From the comparison of these curves, we can see that the nonlinear permeability has little effect on the axial reaction and lateral displacement in the unconfined compression experiment, but from the literature, we can know that, The nonlinear permeability allows the axial reaction to exhibit a distinct nonlinear phase[8], so strain-dependent permeability plays an important role, but its effect can not be manifested in unconfined compression experiments, Due to this experimental mode, the flow of interstitial fluid is relatively easy to perform in confined compression experiments, and its strain is also small [9].

As can be seen from Figs.16 and 17, the fibiril-reinforced porous elasticity model can reflect the change in volume of the articular cartilage during compression, but it does not reflect the change in axial reaction force, which we attribute to the model without considering articular cartilage Viscoelasticity of the solid matrix itself, that is, its intrinsic viscoelasticity in the mechanical behavior of cartilage also plays an important role. Nevertheless, it can be seen that the viscoelastic behavior of the model is still significantly enhanced in comparison to the linear elastic biphasic porous elastic model.

\section{Conclusion}

In this paper, the method of optimization of parameter inversion is introduced, and the main steps of optimizing inversion are realized. It is not possible to invert the parameters of the spring-based fibiril-reinforced model in this way, but instead uses the heuristic Parameters.

In this chapter, we introduce the optimized inversion algorithm for obtaining the parameters of cartilage material, and invert the parameter values of the material by means of heuristics. By comparison, it can not explain the relaxation process of the axial reaction force simultaneously with the fibiril-reinforced porous elastic model. Lateral displacement, that is, the model has obvious deficiencies in explaining the experimental phenomena, such as the two-phase porous viscoelastic model and the fibiril-reinforced porous viscoelastic model. 


\section{References}

1. M. R. Disilvestro, J. K. Suh. A cross-validation of the biphasic poroviscoelastic model of articular cartilage in unconfined compression, indentation, and confined compression[J]. Journal of biomechanics. 2001, 34(4): 519-526.

2. M. R. Disilvestro, Q. Zhu, M. Wong, et al. Biphasic poroviscoelastic simulation of The unconfined compression of articular cartilage: I-Simultaneous prediction of reaction force and lateral displacement[J]. Journal of biomechanical engineering. 2001, 123: 191-197.

3. W. Wilson, C. C. Van. Donkelaar, B. Van Rietbergen, et al. Stresses in the local collagen network of articular cartilage: a poroviscoelastic fibril-reinforced finite element study[J]. Journal of Biomechanics. 2004, 37(3): 357-366.

4. L. P. Li, J. Soulhat, M. D. Buschmann, et al. Nonlinear analysis of cartilage in unconfined ramp compression using a fibril reinforced poroelastic model[J]. Clinical Biomechanics. 1999, 14(9): 673-682.

5. R. K. Korhonen, M. S. Laasanen. Fibril-reinforced poroelastic model predicts specifically mechanical behavior of normal, proteoglycan depleted and collagen degraded articular cartilage[J]. Journal of Biomechanics. 2003, 36(9): 1373-1379.

6. M. Fortin, J. Soulhat, A. Shirazi-Adl, et al. Unconfined compression of articular cartilage: nonlinear behavior and comparison with a fibril-reinforced biphasic model[J]. Journal of biomechanical engineering. 2000, 122: 189.

7. M. Wong, M. Ponticiello, V. Kovanen, et al. Volumetric changes of articular cartilage during stress relaxation in unconfined compression[J]. Journal of biomechanics. 2000, 33(9): 1049-1054.

8. J. Z. Wu, W. Herzog, M. Epstein. Evaluation of the finite element software ABAQUS for biomechanical modelling of biphasic tissues [J]. Journal of biomechanics. 1997, 31(2): 165-169.

9. Cg. L. W. M. V. Armstrong. An analysis of the unconfined compression of articular cartilage [Z]. 1984. 